\title{
ABDOMINAL SECTION
}

FOR

\section{INTESTINAL OBSTRUCTION DUE TO MULTIPLE HYDATID CYSTS.}

\author{
RECOVERY.
}

BY

J. HUTCHINSON, Jun., F.R.C.S., ASSISTANT SURGEON TO THE LONDON HOSPITAL; SURGEON TO OUT-PATIENTS AT THE LOCK HOSPITAL.

Received November 14th, 1893-Read January 9th, 1894.

THE subperitoneal areolar tissue is a not infrequent site for the occurrence of hydatid cysts, and it is a wellrecognised fact that, in this position, they may exist for very many years without giving rise to any symptoms of note (in one recorded case for thirty-five years). The recto-vesical, or Douglas's pouch, would appear to be the region of the abdomen most commonly involved next to the liver; and even in the former, where the cysts are in immediate proximity to the rectum, they very rarely inflame and suppurate or give rise to trouble, except by their bulk and pressure on the pelvic viscera. M. Tuffier ${ }^{1}$

1 'Cinquième Congrès de Chirurgie,' p. 569. 
has collected no less than seventy-four records of cases of hydatid tumours in this region. The interest of the case now reported lies mainly in the fact that intestinal obstruction was produced by three old hydatid cysts and was completely relieved by their removal. There is good reason for thinking that the cysts had existed within the patient's abdomen for no less than twenty-three years; and they well exhibit the remarkable thickness to which the walls of such old cysts may attain. The case is of further interest from the fact that there were only three isolated hydatid tumours in the abdomen, although one was in reality composed of three distinct cysts joined together and in part calcified. Their hardness was greater than that of any tumour except an exostosis, and it may readily be supposed that no correct diagnosis could be made of the cause of obstruction until the abdomen was opened, and that even then at first some doubt was felt as to their nature. The cysts were not directly adherent to the intestine, but were developed in the mesentery.

The patient was a tall thin man aged 53, who had been a gardener all his life. On September 17th, 1893, he was suddenly seized with sickness and abdominal pain; the vomiting continued until his admission to the London Hospital on the 20th. Constipation was present during this time, and the vomiting and pain became more severe. The patient could ascribe the onset to no error of diet or other cause; he had a lobulated tumour in the middle line above the umbilicus some three inches in diameter, and the doctor who attended him suspected this to be a strangulated ventral hernia, and sent him up to the hospital with this diagnosis.

However, on examination, I was sure that the lump was only a protrusion of subperitoneal fat through the linea alba-a " fatty hernia," -and in no way accounted for his symptoms. There was but little in the previous history to throw light on the case ; he had always enjoyed fair health, with the exception of a few "bilious attacks" and the occurrence of ascites at the age of thirty. He was 
then tapped, and after this operation the fluid did not reappear. We may with some confidence ascribe the occurrence of the ascites to the development of the hydatid cysts. The condition of the patient on admission was briefly as follows :-Abdomen somewhat distended, face drawn and anxious, pulse 80 , urine acid and containing no albumen. He was repeatedly sick, and the vomited matter was sourand semi-feculent. He was much troubled with hiccough. A free enema only brought away a little clay-coloured fæces. $\mathrm{He}$ complained much of pain, especially in the upper part of the abdomen.

Per rectum a hard rounded tumour about the size of a cricket-ball could be felt in the pelvis, and with some difficulty a second one, considerably smaller, could be felt through the abdominal wall below and to the right of the umbilicus.

Abdominal exploration was advised, and being at once agreed to by the patient, was performed on the evening of the third day after the onset of symptoms of intestinal obstruction.

An incision below the umbilicus enabled me to feel distinctly the first of the hydatid tumours, lying amongst distended coils of small intestine. It was of stony hardness, and seemed to be bound down to the peritoneum lining the back of the abdomen; however, after a time I succeeded in moving it forwards towards the wound, and then found it presented a strikingly white surface, and that it was probably an old hydatid cyst.

The extraordinary hardness made it almost certain that there would be little trouble from hæmorrhage if it were carefully shelled out from the overlying peritoneum, and this proved to be the case, one or two ligatures only being required. After removal of this first tumour a second one was detected just above the brim of the pelvis, and a third, the largest, lay in that cavity. They were dealt with in the same way by peeling off the peritoneum with forceps and the fingers, mainly the latter-necessarily a tedious process, but one attended by no bleeding worth mentioning. Theo- 
retically it would have been well to sew up the rents in the peritoneum, but practically this was impossible, owing to the distended intestine. After satisfying myself that all bleeding had ceased, some warm boracic solution was used to wash the cavity, the parietal wound in the peritoneum was sewn up by a continuous catgut suture, and the muscles and skin by interrupted silkworm-gut sutures. The stomach was washed out, about half a pint of very offensive brown fluid being removed. The symptoms of obstruction were completely relieved by the operation, but for a week the patient was restless and troublesome, being in a curious condition of melancholia alternating with a kind of delirium. His natural temperament was a melancholic one. The wound healed well but for a small abscess in the abdominal wall, due no doubt to inoculation by the patient's fingers, which he would insert under the dressings unless closely watched. There was some diarrhœa for a week or two, but no symptom pointing to peritonitis. He slowly regained strength, and left the hospital at the end of five weeks.

The description of the tumours was as follows:

The smallest of the cysts measured two inches by one and a half, was oval in shape, and, when the patient was under the anæsthetic, could be felt through the anterior abdominal wall ; it was placed with its long axis transversely. The second one lay just below it, and was more deeply situated, measuring nearly four inches in length, irregularly lobulated and extremely hard, owing to calcareous degeneration of its wall. The third was pyriform, and lay between the bladder and the rectum (through the latter it had been easily felt); it was freely movable although covered with peritoneum, and shelled out easily.

One striking peculiarity of all of the cysts was the great thickness of their walls-tough and laminated on section, looking like cartilage on their surface ; they were about one third of an inch thick. On the inner side was the mother cyst with hundreds of daughter cysts and brood-capsules. The daughter cysts varied in size from 
that of small shot up to that of one inch in diameter, and were closely packed together. In the fluid hooklets of the echinococcus were found, and it was fairly easy to recognise here and there the complete parasite.

The median cyst was composed of three, all with thick walls containing calcareous matter and each containing many daughter cysts.

Little allusion has yet been made to one interesting point-how these hydatid tumours, which had probably existed in the patient's abdomen for some twenty-three years, had suddenly caused intestinal obstruction. The operation did not clearly show exactly where the obstruction was, but it was certainly in the small intestine, and I have little doubt that it was due to the largest of the three hydatids becoming fixed in the pelvis, and thus exercising traction on the mesentery in which they lay. It was almost certainly a mechanical obstruction, there being no trace of inflammation around the tumours. No other hydatids were found within the abdomen, and the case is of interest from this point of view, it being rare for peritoneal hydatid cysts to occur except in a widely disseminated manner. Those who have had much experience of echinococcus disease in Australia tell me that such extremely thick-walled cysts are very rare indeed. Equally uncommon is it for intestinal obstruction to owe its cause to hydatids.

It may once again be pointed out that, with regard to the cysts obstructing the intestine, they formed a sort of chain some eight inches long, reaching from just to the right of the umbilicus down to the bottom of the rectovesical pouch. They were certainly embedded in the mesentery and not in the great omentum.

In the 'Pathological 'Transactions' (vol. v, p. 302) is narrated the case of a middle-aged woman who died with symptoms of acute intestinal obstruction lasting for six days. No operation was performed. At the post-mortem an hydatid cyst the size of a large orange was found in the meso-rectum, causing occlusion of the rectum and 
sloughing of its coats by pressure. A number of other cysts were found in the peritoneum of the intestines, uterus, and Fallopian tubes. There were no adhesions. Most of the cysts contained daughter ones. During life one of the cystic tumours could be felt near the umbilicus.

In 1871 Sir Spencer Wells operated on a woman with multiple hydatids scattered about the peritoneum of the intestine and omentum. Only a partial removal was effected, and the patient died within a year, after an illness characterised by vomiting and purging. ${ }^{1}$

I have collected a few other accounts of cases in which hydatid cysts caused death by pressing upon one or other part of the intestinal tract.

In conclusion, I would draw attention to the fact that the presence of a fatty hernia just above the umbilicus led to an erroneous diagnosis of the cause of the intestinal symptoms, probably a not very infrequent source of error. Whether occurring in the linea alba, the femoral canal, or the inguinal region, these protrusions of subperitoneal fat are apt closely to simulate true herniæ. In two papers published in the 'Pathological Society's Transactions' I have collected several instances, and since then have known of other cases in which symptoms of intestinal obstruction occurring in subjects possessing a fatty hernia have been wrongly suspected to be due to the latter. It is a fallacy to be guarded against, especially since it is as a rule both irksome and useless to attempt to retain a fatty hernia by means of truss-pressure. Nevertheless it may be admitted that sometimes the protrusion of the subperitoneal fat draws with it a pouch of peritoneum and so may lead to the production of a true hernia. In the case just described I examined the abdominal wall opposite the supposed hernia from within, and found no peritoneal protrusion.

1 'Path. Soc. Trans.,' vol. xvii, p. 278.

(For report of the discussion on this paper, see 'Proceedings of the Royal Medical and Chirurgical Society,' Third Series, vol. vi. p. 41.) 


\section{DESCRIPTION OF PLATE III.}

\section{Abdominal Section for Intestinal Obstruction due to Multiple \\ Hydatid Cysts (J. Hutchinson, Jun.).}

Case of multiple hydatid cysts causing acute intestinal obstruction. The cysts are represented in the order they were found in the abdomen, the smallest high up, the largest (Fig. 3) in the pelvis.

FIGs. 1 and 2 represent sectional views, and show many daughter cysts within an extremely thick and tough capsule. To the left of Fig. 2 is shown a small secondary cyst which was calcified.

Fig. 3 represents the outer surface of the largest cyst with some peritoneum and vessels attached to it. All the cysts lay in the mesentery. 


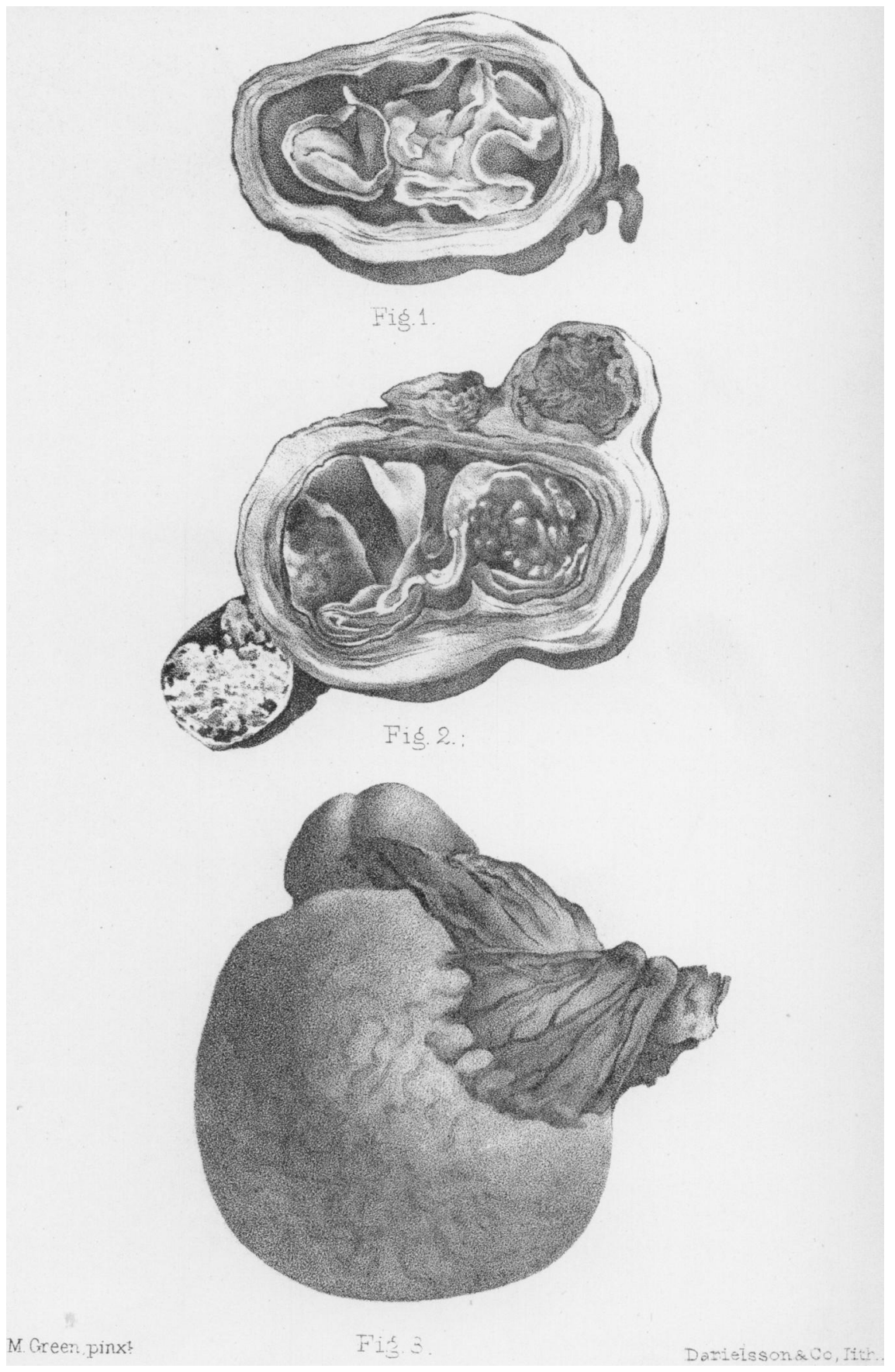

